Network Working Group

Request for Comments: 4328

Updates: 3471

Category: Standards Track
D. Papadimitriou, Ed.

Alcatel

January 2006

\title{
Generalized Multi-Protocol Label Switching (GMPLS) \\ Signaling Extensions for G.709 Optical Transport Networks Control
}

Status of This Memo

This document specifies an Internet standards track protocol for the Internet community, and requests discussion and suggestions for improvements. Please refer to the current edition of the "Internet Official Protocol Standards" (STD 1) for the standardization state and status of this protocol. Distribution of this memo is unlimited.

Copyright Notice

Copyright (C) The Internet Society (2006) .

Abstract

This document is a companion to the Generalized Multi-Protocol Label Switching (GMPLS) signaling documents. It describes the technologyspecific information needed to extend GMPLS signaling to control Optical Transport Networks (OTN); it also includes the so-called pre-OTN developments.

Table of Contents

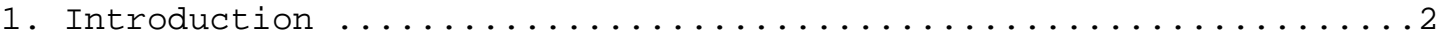

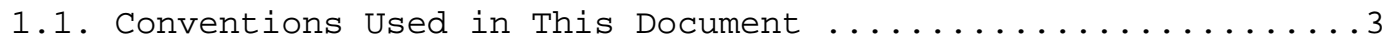

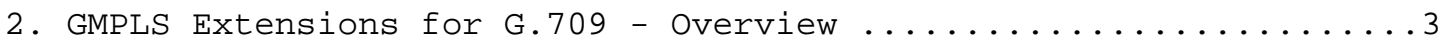

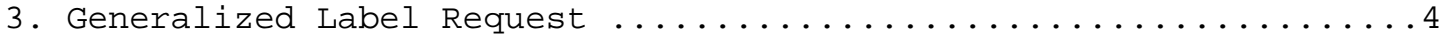

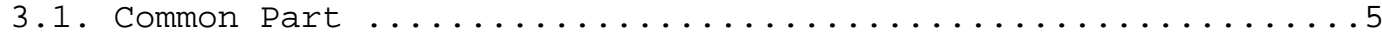

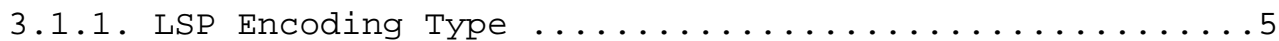

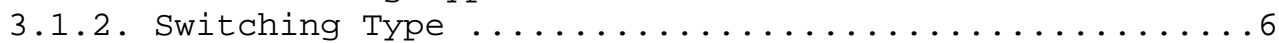

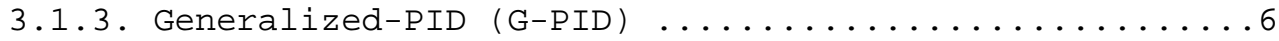

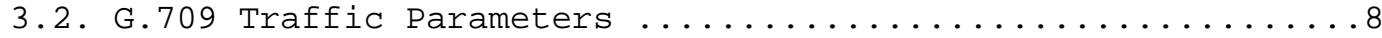

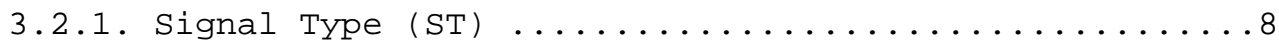

3.2.2. Number of Multiplexed Components (NMC) ..........9

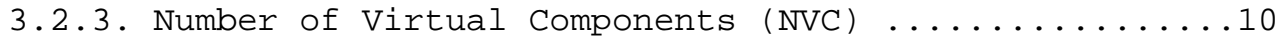

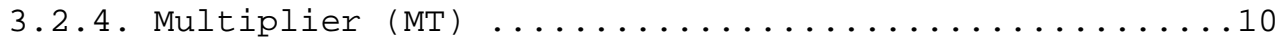

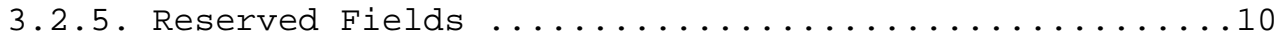

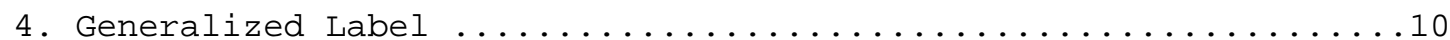

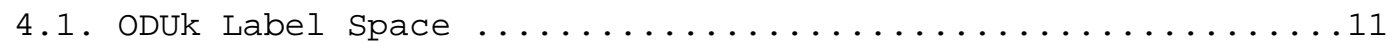

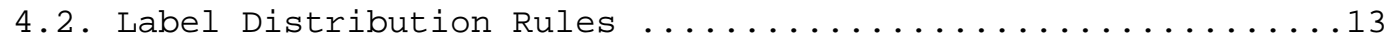




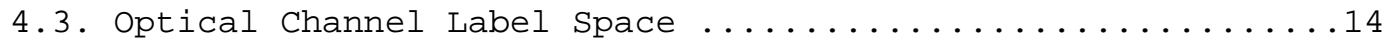

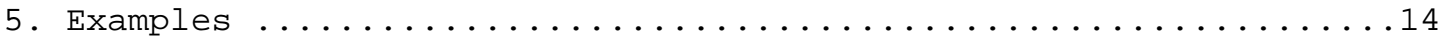

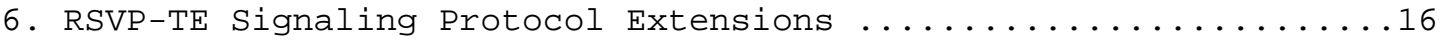

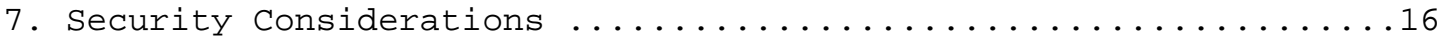

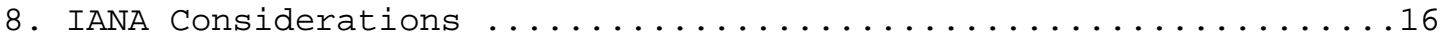

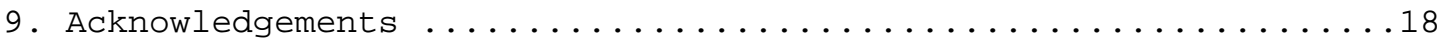

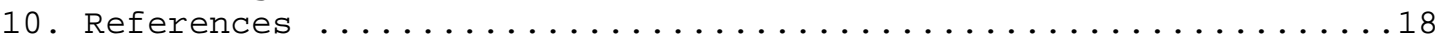

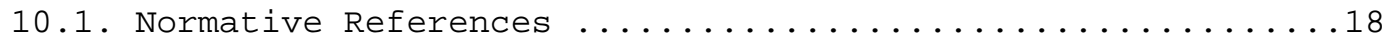

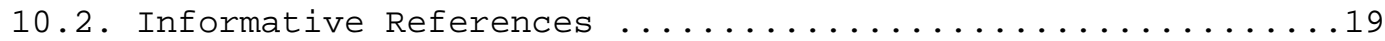

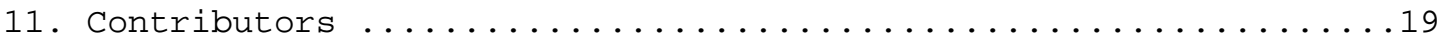

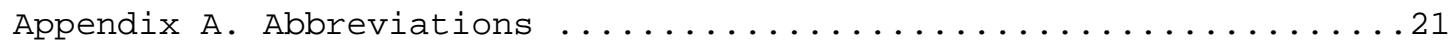

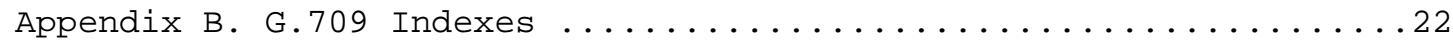

1. Introduction

Generalized Multi-Protocol Label Switching (GMPLS) [RFC3945] extends MPLS from supporting Packet Switching Capable (PSC) interfaces and switching to include support of four new classes of interfaces and switching: Layer-2 Switching (L2SC), Time-Division Multiplex (TDM), Lambda Switch (LSC), and Fiber-Switch (FSC) Capable. A functional description of the extensions to MPLS signaling that are needed to support these new classes of interfaces and switching is provided in [RFC3471]. [RFC3473] describes the RSVP-TE-specific formats and mechanisms needed to support all four classes of interfaces.

This document presents the technology details that are specific to G.709 Optical Transport Networks (OTN) as specified in the ITU-T G.709 recommendation [ITUT-G709] (and referenced documents), including pre-OTN developments. Per [RFC3471], G.709 technologyspecific parameters are carried through the signaling protocol in dedicated traffic parameter objects.

The G.709 traffic parameters defined hereafter (see Section 3.2) MUST be used when the label is encoded as defined in this document. Moreover, the label MUST be encoded as defined in section 4 when these G.709 traffic parameters are used.

In the context of this memo, by pre-OTN developments, one refers to Optical Channel, Digital Wrapper and Forward Error Correction (FEC) solutions that are not fully G.709 compliant. Details concerning pre-OTN Synchronous Optical Network (SONET)/Synchronous Digital Hierarchy (SDH) based solutions including Section/Regenerator section overhead ( $\mathrm{SOH} / \mathrm{RSOH}$ ) and Line/Multiplex Section overhead (LOH/MSOH) transparency are covered in [RFC3946]. 


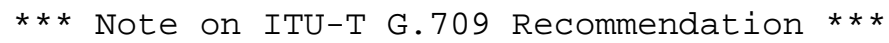

The views on the ITU-T G.709 OTN Recommendation presented in this document are intentionally restricted to the GMPLS perspective within the IETF CCAMP WG context. Hence, the objective of this document is not to replicate the content of the ITU-T OTN recommendations. Therefore, readers interested in more details concerning the corresponding technologies are strongly invited to consult the corresponding ITU-T documents (also referenced in this memo).

1.1. Conventions Used in This Document

The key words "MUST", "MUST NOT", "REQUIRED", "SHALL", "SHALL NOT", "SHOULD", "SHOULD NOT", "RECOMMENDED", "MAY", and "OPTIONAL" in this document are to be interpreted as described in [RFC2119].

In addition, the reader is assumed to be familiar with the terminology used in ITU-T [ITUT-G709], as well as [RFC3471] and [RFC3473]. Abbreviations used in this document are detailed in Appendix 1 .

2. GMPLS Extensions for G.709 - Overview

[ITUT-G709] defines several networking layers constituting the optical transport hierarchy:

- with full functionality:

- Optical Transmission Section (OTS)

- Optical Multiplex Section (OMS)

- Optical Channel (OCh)

- with reduced functionality:

- Optical Physical Section (OPS)

- Optical Channel with reduced functionality (ochr)

It also defines two layers constituting the digital transport hierarchy:

- Optical Channel Transport Unit (OTUk)

- Optical Channel Data Unit (ODUk)

However, only the OCh and the ODUk layers are defined as switching layers. Both OCh (but not OChr) and ODUk layers include the overhead for supervision and management. The och overhead is transported in a non-associated manner (also referred to as the non-associated overhead naOH) in the Optical Transport Module (OTM) Overhead Signal (OOS), together with the OTS and OMS non-associated overhead. The oOS is transported via a dedicated wavelength, referred to as the Optical Supervisory Channel (OSC). It should be noticed that the 
naOH is only functionally specified and as such, it is open to vendor-specific solutions. The ODUk overhead is transported in an associated manner as part of the digital ODUk frame.

As described in [ITUT-G709], in addition to the support of ODUk mapping into OTUk $(\mathrm{k}=1,2,3), \mathrm{G} .709$ supports ODUk multiplexing . It refers to the multiplexing of $\operatorname{ODUj}(j=1,2)$ into an ODUk $(k>j)$ signal, in particular:

- ODU1 into ODU2 multiplexing

- ODU1 into ODU3 multiplexing

- ODU2 into ODU3 multiplexing

- ODU1 and ODU2 into ODU3 multiplexing

Adapting GMPLS to control G.709 OTN can be achieved by creating:

- a Digital Path layer, by extending the previously defined "Digital Wrapper" in [RFC3471] corresponding to the ODUk (digital) path layer.

- an Optical Path layer, by extending the "Lambda" concept (defined in [RFC3471]) to the OCh (optical) path layer.

- a label space structure, by considering a tree whose root is an OTUk signal and leaves the ODUj signals $(\mathrm{k}>=j)$; enabling the identification of the exact position of a particular ODUj signal in an ODUk multiplexing structure.

Thus, the GMPLS signaling extensions for G.709 need to cover the Generalized Label Request, the Generalized Label as well as the specific technology dependent objects included in the so-called traffic parameters as specified in [RFC3946] for SONET/SDH networks. Moreover, because multiplexing in the digital domain (such as ODUk multiplexing) has been specified in the amended version of the G.709 ITU-T recommendation (October 2001), this document also proposes a label space definition suitable for that purpose. Notice also that one uses the G.709 ODUk (i.e., Digital Path) and OCh (i.e., Optical Path) layers directly in order to define the corresponding label spaces.

3. Generalized Label Request

The Generalized Label Request, as defined in [RFC3471], includes a common part (i.e., used for any switching technology) and a technology dependent part (i.e., the traffic parameters). In this section, both parts are extended to accommodate GMPLS Signaling to the G.709 transport plane recommendation (see [ITUT-G709]). 


\subsection{Common Part}

As defined in [RFC3471], the LSP Encoding Type, the Switching Type and the Generalized Protocol Identifier (Generalized-PID) constitute the common part of the Generalized Label Request. The encoding of the RSVP-TE GENERALIZED_LABEL_REQUEST object is specified in [RFC3473] Section 2.1.

As mentioned above, this document extends the LSP Encoding Type, the Switching Type, and G-PID (Generalized-PID) values to accommodate G.709 Recommendation [ITUT-G709].

\subsubsection{LSP Encoding Type}

Because G.709 Recommendation defines two networking layers (ODUk layers and OCh layer), the LSP Encoding Type code-points can reflect these two layers defined in [RFC3471] Section 3.1 as "Digital Wrapper" and "Lambda" code. The LSP Encoding Type is specified per networking layer or, more precisely, per group of functional networking layers: the ODUk layers and the och layer.

Therefore, an additional LSP Encoding Type code-point for the G.709 Digital Path layer is defined; it enlarges the existing "Digital Wrapper" code-point defined in [RFC3471]. The former MUST be generated when the interface or tunnel on which the traffic will be transmitted supports G.709 compliant Digital Path layer encoding. The latter MUST only be used for non-G.709 compliant Digital Wrapper layer(s) encoding. A transit or an egress node (receiving a Path message containing a GENERALIZED_LABEL_REQUEST object) MUST generate a PathErr message, with a "Routing problem/Unsupported Encoding" indication, if the requested LSP Encoding Type cannot be supported on the corresponding incoming interface.

In the same way, an additional LSP Encoding Type code-point for the G.709 Optical Channel layer is defined; it enlarges the existing "Lambda" code-point defined in [RFC3471]. The former MUST be generated when the interface or tunnel on which the traffic will be transmitted supports G.709-compliant Optical Channel layer encoding. The latter MUST only be used for non-G.709 compliant Lambda layer(s) encoding. A transit or an egress node (receiving a Path message that contains a GENERALIZED_LABEL_REQUEST object) MUST generate a PathErr message with a "Routing problem/Unsupported Encoding" indication, if the requested LSP Encoding Type cannot be supported on the corresponding incoming interface. 
Consequently, the following additional code-points for the LSP Encoding Type are defined:

$\begin{array}{lr}\text { Value } & \text { Type } \\ ----- & ---- \\ 12 & \text { G.709 ODUk (Digital Path) } \\ 13 & \text { G.709 Optical Channel }\end{array}$

Moreover, the code-point for the G.709 Optical Channel (OCh) layer will indicate the requested capability of an end-system to use the G.709 non-associated overhead (naOH), i.e., the OTM Overhead Signal (OOS) multiplexed into the OTM-n.m interface signal.

\subsubsection{Switching Type}

The Switching Type indicates the type of switching that should be performed at the termination of a particular link (see [RFC4202]).

No additional Switching Type values are to be considered in order to accommodate G.709 switching types, because an ODUk switching (and thus LSPS) belongs to the TDM class, while an OCh switching (and thus LSPS) belong to the Lambda class (i.e., LSC).

Intermediate and egress nodes MUST verify that the value indicated in the Switching Type field is supported on the corresponding incoming interface. If the requested value can not be supported, the node MUST generate a PathErr message with a "Routing problem/Switching Type" indication.

\subsubsection{Generalized-PID (G-PID)}

The G-PID (16 bits field), as defined in [RFC3471], identifies the payload carried by an LSP, i.e., an identifier of the client layer of that LSP. This identifier is used by the endpoints of the G.709 LSP.

The G-PID can take one of the following values when the client payload is transported over the Digital Path layer, in addition to the payload identifiers defined in [RFC3471]:

- CBRa: asynchronous Constant Bit Rate (i.e., mapping of STM-16/OC48, STM-64/OC-192 and STM-256/OC-768)

- CBRb: bit synchronous Constant Bit Rate (i.e., mapping of STM16/OC-48, STM-64/OC-192 and STM-256/OC-768)

- ATM: mapping at $2.5,10$ and 40 Gbps

- BSOT: non-specific client Bit Stream with Octet Timing (i.e., Mapping of $2.5,10$ and 40 Gbps Bit Stream)

- BSNT: non-specific client Bit Stream without Octet Timing (i.e., Mapping of $2.5,10$ and 40 Gbps Bit Stream) 
- ODUk: transport of Digital Paths at 2.5, 10 and 40 Gbps

- ESCON: Enterprise Systems Connection

- FICON: Fiber Connection

The G-PID can take one of the following values when the client payload is transported over the optical Channel layer, in addition to the payload identifiers defined in [RFC3471]:

- CBR: Constant Bit Rate (i.e., mapping of STM-16/OC-48, STM-64/OC192 and STM-256/OC-768)

- OTUk/OTUkV: transport of Digital Section at 2.5, 10 and 40 Gbps

Also, when client payloads such as Ethernet MAC/PHY and IP/PPP are encapsulated through the Generic Framing Procedure (GFP), as described in ITU-T G.7041, dedicated G-PID values are defined.

In order to include pre-OTN developments, the G-PID field can take one of the values (currently defined in [RFC3471]) when the following client payloads are transported over a so-called lambda LSP:

- Ethernet PHY (1 Gbps and 10 Gbps)

- Fiber Channel

The following table summarizes the G-PID with respect to the LSP Encoding Type:

\begin{tabular}{|c|c|c|}
\hline Value & G-PID Type & LSP Encoding Type \\
\hline 47 & G 709 & (with $k$ \\
\hline 48 & G.709 OTUk (v) & G.709 OCh \\
\hline & & ODUk mapped into OTUk (v) \\
\hline 49 & $\mathrm{CBR} / \mathrm{CBRa}$ & G.709 ODUk, G.709 OCh \\
\hline 50 & CBRb & G.709 ODUk \\
\hline 51 & $\mathrm{BSOT}$ & G.709 ODUk \\
\hline 52 & $\mathrm{BSNT}$ & G.709 ODUk \\
\hline 53 & IP /PPP (GFP) & G.709 ODUk \\
\hline 54 & Ethernet MAC (framed GFP) & G.709 ODUk \\
\hline 55 & Ethernet PHY (transparent GFP) & G.709 ODUk (and SDH) \\
\hline 56 & ESCON & G.709 ODUk, Lambda, Fiber \\
\hline 57 & FICON & G.709 ODUk, Lambda, Fiber \\
\hline 58 & Fiber Channel & G.709 ODUk, Lambda, Fiber \\
\hline
\end{tabular}

Note: Values 49 and 50 include mapping of SDH. 
The following table summarizes the update of the G-PID values defined in [RFC3471]:

$\begin{array}{lll}\text { Value } & \text { G-PID Type } & \text { LSP Encoding Type } \\ ---- & \text {-------- } & ------------ \\ 32 & \text { ATM Mapping } & \text { SDH, G.709 ODUk } \\ 33 & \text { Ethernet PHY } & \text { SDH, G.709 OCh, Lambda, Fiber } \\ 34 & \text { Sonet/SDH } & \text { G.709 OCh, Lambda, Fiber } \\ 35 & \text { Reserved (SONET Dep.) } & \text { G.709 OCh, Lambda, Fiber }\end{array}$

\subsection{G.709 Traffic Parameters}

When G.709 Digital Path Layer or G.709 Optical Channel Layer is specified in the LSP Encoding Type field, the information referred to as technology dependent (or simply traffic parameters) is carried additionally to the one included in the Generalized Label Request.

The G.709 traffic parameters are defined as follows:

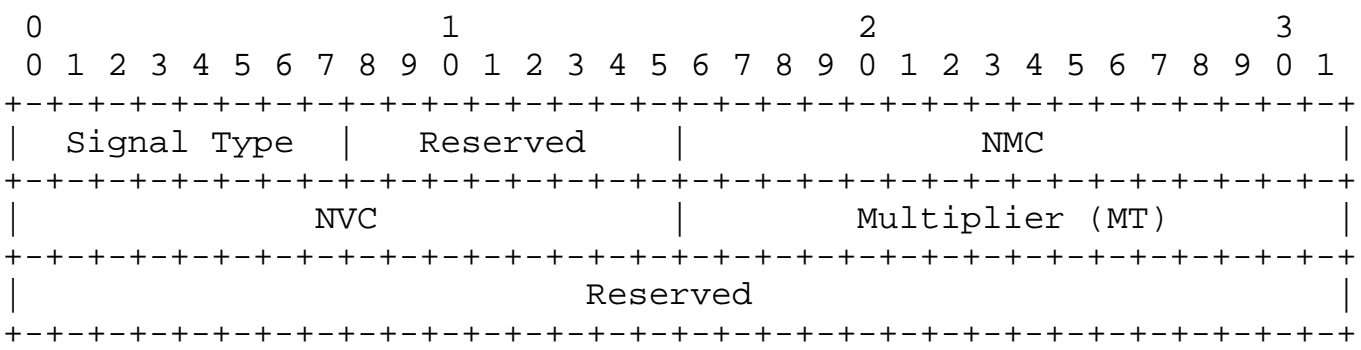

In this frame, NMC stands for Number of Multiplexed Components, NVC for Number of Virtual Components, and MT for Multiplier. Each of these fields is tailored to support G.709 LSP requests.

The RSVP-TE encoding of the G.709 traffic-parameters is detailed in Section 6 .

\subsubsection{Signal Type (ST)}

This field ( 8 bits) indicates the type of G.709 Elementary Signal that comprises the requested LSP. The permitted values are:

$\begin{array}{cl}\text { Value } & \text { Type } \\ ---- & ---- \\ 0 & \text { Not significant } \\ 1 & \text { ODU1 (i.e., } 2.5 \text { Gbps) } \\ 2 & \text { ODU2 (i.e., } 10 \text { Gbps) } \\ 3 & \text { ODU3 (i.e., } 40 \text { Gbps) } \\ 4 & \text { Reserved (for future use) }\end{array}$




$\begin{array}{ll}5 & \text { Reserved (for future use) } \\ 6 & \text { OCh at } 2.5 \text { Gbps } \\ 7 & \text { OCh at } 10 \text { Gbps } \\ 8 & \text { OCh at } 40 \text { Gbps } \\ 9-255 & \text { Reserved (for future use) }\end{array}$

The value of the Signal Type field depends on LSP Encoding Type value defined in section 3.1.1 and [RFC3471]:

- if the LSP Encoding Type value is the G.709 Digital Path layer, then the valid values are the oDUk signals ( $\mathrm{k}=1,2$ or 3 ).

- if the LSP Encoding Type value is the G.709 Optical Channel layer, then the valid values are the OCh at 2.5, 10, or 40 Gbps.

- if the LSP Encoding Type is "Lambda" (which includes the pre-OTN Optical Channel layer) then the valid value is irrelevant (Signal Type $=0$ ).

- if the LSP Encoding Type is "Digital Wrapper", then the valid value is irrelevant (Signal Type $=0$ ).

Several transforms can be sequentially applied on the Elementary Signal to build the Final Signal that is actually requested for the LSP. Each transform application is optional and must be ignored if zero; this does not include the Multiplier (MT), which cannot be zero and must be ignored if equal to one. Transforms must be applied strictly in the following order:

- First, virtual concatenation (by using the NVC field) can be optionally applied directly on the Elementary signal to form a Composed Signal

- Second, a multiplication (by using the Multiplier field) can be optionally applied, either directly on the Elementary Signal, or on the virtually concatenated signal obtained from the first phase. The resulting signal is referred to as Final Signal.

\subsubsection{Number of Multiplexed Components (NMC)}

The NMC field (16 bits) indicates the number of ODU tributary slots used by an ODUj when multiplexed into an ODUk $(k>j)$ for the requested LSP. This field is not applicable when an ODUk is mapped into an OTUk and irrelevant at the Optical Channel layer. In both cases, it MUST be set to zero ( $\mathrm{NMC}=0)$ when sent and should be ignored when received.

When applied at the Digital Path layer, in particular for oDU2 connections multiplexed into one ODU3 payload, the NMC field specifies the number of individual tributary slots $(\mathrm{NMC}=4)$ that constitute the requested connection. These components are still processed within the context of a single connection entity. For all 
other currently defined multiplexing cases (see Section 2), the NMC field is set to 1 .

3.2.3. Number of Virtual Components (NVC)

The NVC field (16 bits) is dedicated to ODUk virtual concatenation (i.e., ODUk Inverse Multiplexing) purposes. It indicates the number of ODU1, ODU2, or ODU3 Elementary Signals that are requested to be virtually concatenated to form an ODUk-Xv signal. By definition, these signals MUST be of the same type.

This field is set to 0 (default value) to indicate that no virtual concatenation is requested.

Note that the current usage of this field only applies for G.709 ODUk LSPs, i.e., values greater than zero, are only acceptable for oDUk Signal Types. Therefore, it MUST be set to zero $($ NVC $=0)$, and should be ignored when received, when a G.709 OCh LSP is requested.

\subsubsection{Multiplier (MT)}

The Multiplier field (16 bits) indicates the number of identical Elementary Signals or Composed Signals that are requested for the LSP, i.e., that form the Final Signal. A Composed Signal is the resulting signal from the application of the NMC and NVC fields to an elementary Signal Type. GMPLS signaling currently implies that all the Composed Signals must be part of the same LSP.

This field is set to one (default value) to indicate that exactly one instance of a signal is being requested. Intermediate and egress nodes MUST verify that the node itself and the interfaces on which the LSP will be established can support the requested multiplier value. If the requested values cannot be supported, the receiver node MUST generate a PathErr message (see Section 6).

Zero is an invalid value for the MT field. If received, the node MUST generate a PathErr message (see Section 6).

\subsubsection{Reserved Fields}

The reserved fields ( 8 bits in row 1 and 32 bits in row 3 ) are dedicated for future use. Reserved bits SHOULD be set to zero when sent and MUST be ignored when received.

4. Generalized Label

This section describes the Generalized Label value space for Digital Paths and Optical Channels. The Generalized Label is defined in 
[RFC3471]. The format of the corresponding RSVP-TE GENERALIZED_LABEL object is specified in [RFC3473] Section 2.3.

The label distribution rules detailed in section 4.2 follow (when applicable) the ones defined in [RFC3946].

\subsection{ODUk Label Space}

At the Digital Path layer (i.e., ODUk layers), G.709 defines three different client payload bit rates. An Optical Data Unit (ODU) frame has been defined for each of these bit rates. ODUk refers to the frame at bit rate $\mathrm{k}$, where $\mathrm{k}=1$ (for $2.5 \mathrm{Gbps}$ ), 2 (for $10 \mathrm{Gbps}$ ), or 3 (for 40 Gbps).

In addition to the support of ODUk mapping into OTUk, the G.709 label space supports the sub-levels of ODUk multiplexing. ODUk multiplexing refers to multiplexing of ODUj $(j=1,2)$ into an ODUk $(k>j)$, in particular:

- ODU1 into ODU2 multiplexing

- ODU1 into ODU3 multiplexing

- ODU2 into ODU3 multiplexing

- ODU1 and ODU2 into ODU3 multiplexing

More precisely, ODUj into ODUk multiplexing ( $\mathrm{k}>\mathrm{j}$ ) is defined when an ODUj is multiplexed into an ODUk Tributary Unit Group (i.e., an ODTUG constituted by ODU tributary slots) that is mapped into an OPUk. The resulting OPUk is mapped into an ODUk, and the ODUk is mapped into an OTUk.

Therefore, the label space structure is a tree whose root is an OTUk signal and whose leaves are the ODUj signals ( $k>=j)$ that can be transported via the tributary slots and switched between these slots. A G.709 Digital Path layer label identifies the exact position of a particular ODUj signal in an ODUk multiplexing structure.

The G.709 Digital Path Layer label or ODUk label has the following format:

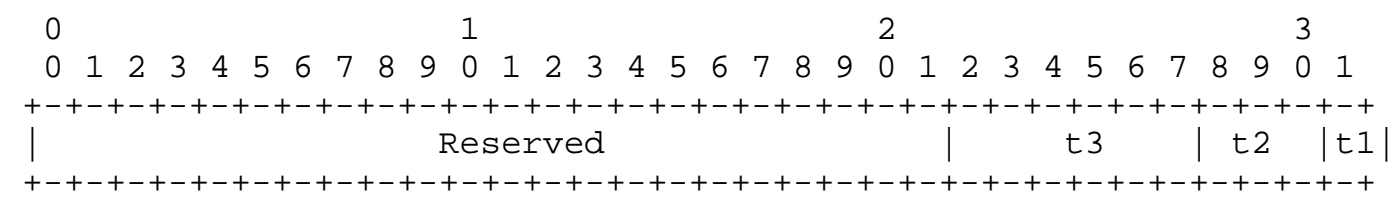

Reserved bits MUST be set to zero when sent and SHOULD be ignored when received. 
The specification of the fields $t 1$, $t 2$, and $t 3$ self-consistently characterizes the ODUk label space. The value space for the $t 1, t 2$, and $t 3$ fields is defined as follows:

1. $t 1$ (1-bit) :

- t1=1 indicates an ODU1 signal.

- t1 is not significant for the other ODUk signal types (i.e., t1 value MUST be set to 0 and ignored).

2. $t 2(3-b i t):$

- t2=1 indicates an ODU2 signal that is not further subdivided.

- t2=[2..5] indicates the tributary slot (t2th-2) used by the ODU1 in an ODTUG2 mapped into an ODU2 (via OPU2).

- t2 is not significant for an ODU3 (i.e., t2 value MUST be set to 0 and ignored).

3. $t 3(6-b i t):$

- t3=1 indicates an ODU3 signal that is not further subdivided.

- $t 3=[2.17]$ indicates the tributary slot (t3th-1) used by the ODU1 in an ODTUG3 mapped into an ODU3 (via OPU3) .

- t3=[18..33] indicates the tributary slot (t3th-17) used by the ODU2 in an ODTUG3 mapped into an ODU3 (via OPU3).

Note: in case of ODU2 into ODU3 multiplexing, 4 labels are required to identify the 4 tributary slots used by the oDU2; these tributary time slots have to be allocated in ascending order.

If the label sub-field value $t[i]=1$ ( $i, j=1,2$ or 3 ) and $t[j]=0$ (j $>i)$, the corresponding ODUk signal ODU[i] is directly mapped into the corresponding OTUk signal $(\mathrm{k}=\mathrm{i})$. This is referred to as the mapping of an ODUk signal into an OTUk of the same order. Therefore, the numbering starts at $1 ;$ zero is used to indicate a non-significant field. A label field equal to zero is an invalid value.

Examples:

- $t 3=0, t 2=0, t 1=1$ indicates an ODU1 mapped into an OTU1

- t3=0, $t 2=1, t 1=0$ indicates an ODU2 mapped into an OTU2

- $t 3=1, t 2=0, t 1=0$ indicates an ODU3 mapped into an OTU3

- $t 3=0, t 2=3, t 1=0$ indicates the ODU1 in the second tributary slot of the ODTUG2 mapped into an ODU2 (via OPU2) mapped into an OTU2

- $t 3=5, t 2=0, t 1=0$ indicates the ODU1 in the fourth tributary slot of the ODTUG3 mapped into an ODU3 (via OPU3) mapped into an oTU3 


\subsection{Label Distribution Rules}

In case of ODUk in OTUk mapping, only one label can appear in the Generalized Label. The unique label is encoded as a single 32-bit label value (as defined in Section 4.1) of the GENERALIZED_LABEL object (Class-Num $=16, \mathrm{C}$-Type $=2)$.

In case of ODUj in ODUk $(k>j)$ multiplexing, the explicit ordered list of the labels in the multiplex is given (this list can be restricted to only one label when $\mathrm{NMC}=1$ ). Each label indicates a component (ODUj tributary slot) of the multiplexed signal. The order of the labels must reflect the order of the ODUj into the multiplex (not the physical order of tributary slots). This ordered list of labels is encoded as a sequence of 32-bit label values (as defined in Section 4.1) of the GENERALIZED_LABEL object (Class-Num = 16, C-Type $=2$ ).

In case of ODUk virtual concatenation, the explicit ordered list of all labels in the concatenation is given. Each label indicates a component of the virtually concatenated signal. The order of the labels must reflect the order of the ODUk to concatenate (not the physical order of time-slots). This representation limits virtual concatenation to remain within a single (component) link. In case of multiplexed virtually concatenated signals, the first set of labels indicates the components (ODUj tributary slots) of the first virtually concatenated signal, the second set of labels indicates the components (ODUj tributary slots) of the second virtually concatenated signal, and so on. This ordered list of labels is encoded as a sequence of 32-bit label values (as defined in section 4.1) of the GENERALIZED_LABEL object (Class-Num = 16, C-Type = 2). In case of ODUk virtual concatenation, the number of label values is determined by the NVC value. Multiplexed oDUk virtual concatenation additionally uses the NMC value to determine the number of labels per set (equal in size).

In case of multiplication (i.e., when using the MT field), the explicit ordered list of all labels taking part in the composed signal is given. The above representation limits multiplication to remain within a single (component) link. In case of multiplication of multiplexed virtually concatenated signals, the first set of labels indicates the components of the first multiplexed virtually concatenated signal, the second set of labels indicates components of the second multiplexed virtually concatenated signal, and so on. This ordered list of labels is encoded as a sequence of 32-bit label values (as defined in Section 4.1) of the GENERALIZED_LABEL object $($ Class-Num $=16, \mathrm{C}-$ Type $=2)$. In case of multiplication of (equal) ODUk virtual concatenated signals, the number of label values per signal is determined by the NVC value. Multiplication of multiplexed 
(equal) ODUk virtual concatenation additionally uses the NMC value to determine the number of labels per set (equal in size).

\subsection{Optical Channel Label Space}

At the Optical Channel layer, the label space must be consistently defined as a flat space whose values reflect the local assignment of och identifiers that correspond to the OTM-n.m sub-interface signals $(m=1,2$ or 3$)$. Note that these identifiers do not cover ochr because the corresponding Connection Function (OChr-CF) between OTMnr.m/OTM-Or.m is not defined in [ITUT-G798].

The OCh label space values are defined by either absolute values (i.e., channel identifiers or Channel ID, also referred to as wavelength identifiers) or relative values (channel spacing, also referred to as inter-wavelength spacing). The latter is strictly confined to a per-port label space, whereas the former could be defined as a local or a global (per node) label space. Such an och label space is applicable to both OTN Optical Channel layer and preOTN Optical Channel layer.

Optical Channel label encoding (and distribution) rules are defined in [RFC3471]. They MUST be used for the Upstream Label, the Suggested Label, and the Generalized Label.

\section{Examples}

The following examples are given in order to illustrate the processing described in the previous sections of this document.

1. ODUk in OTUk mapping: when one ODU1 (ODU2 or ODU3) signal is directly transported in an OTU1 (OTU2 or OTU3), the upstream node requests results simply in an ODU1 (ODU2 or ODU3) signal request.

In such conditions, the downstream node has to return a unique label because the ODU1 (ODU2 or ODU3) is directly mapped into the corresponding OTU1 (OTU2 or OTU3). Because a single ODUk signal is requested (Signal Type $=1,2$ or 3), the downstream node has to return a single ODUk label, which can be, for instance, one of the following when the Signal Type $=1$ :

- $t 3=0, t 2=0, t 1=1$ indicating a single ODU1 mapped into an OTU1

- $t 3=0, t 2=1, t 1=0$ indicating a single ODU2 mapped into an OTU2

- $t 3=1, t 2=0, t 1=0$ indicating a single oDU3 mapped into an OTU3

2. ODU1 into ODUk multiplexing $(k>1)$ : when one ODU1 is multiplexed into the payload of a structured ODU2 (or ODU3), the upstream node requests results simply in an ODU1 signal request. 
In such conditions, the downstream node has to return a unique label because the ODU1 is multiplexed into one ODTUG2 (or ODTUG3). The latter is then mapped into the ODU2 (or ODU3) via OPU2 (or OPU3) and then mapped into the corresponding OTU2 (or OTU3). Because a single ODU1 multiplexed signal is requested (Signal Type $=1$ and $\mathrm{NMC}=1$ ), the downstream node has to return a single ODU1 label, which can take, for instance, one of the following values:

- $t 3=0, t 2=4, t 1=0$ indicates the ODU1 in the third TS of the ODTUG2 - $t 3=2, t 2=0, t 1=0$ indicates the ODU1 in the first TS of the ODTUG 3

- $t 3=7, t 2=0, t 1=0$ indicates the ODU1 in the sixth TS of the ODTUG3

3. ODU2 into ODU3 multiplexing: when one unstructured ODU2 is multiplexed into the payload of a structured ODU3, the upstream node requests results simply in an ODU2 signal request.

In such conditions, the downstream node has to return four labels since the ODU2 is multiplexed into one ODTUG3. The latter is mapped into an ODU3 (via OPU3) and then mapped into an OTU3. Since an ODU2 multiplexed signal is requested (Signal Type $=2$, and NMC $=4)$, the downstream node has to return four ODU labels which can take for instance the following values:

- $t 3=18, t 2=0, t 1=0$ (first part of ODU2 in first TS of ODTUG3)

- $t 3=22$, $t 2=0, t 1=0$ (second part of ODU2 in fifth TS of ODTUG3)

- $t 3=23, t 2=0, t 1=0$ (third part of ODU2 in sixth TS of ODTUG3)

$-t 3=26, t 2=0, t 1=0$ (fourth part of ODU2 in ninth TS of ODTUG3)

4. When a single OCh signal of 40 Gbps is requested (Signal Type $=$ 8), the downstream node must return a single wavelength label as specified in [RFC3471].

5. When requesting multiple ODUk LSP (i.e., with a multiplier (MT) value >1), an explicit list of labels is returned to the requestor node.

When the downstream node receives a request for a $4 \mathrm{x}$ ODU1 signal ( Signal Type $=1, \mathrm{NMC}=1$ and $\mathrm{MT}=4$ ) multiplexed into an ODU3, it returns an ordered list of four labels to the upstream node: the first ODU1 label corresponds to the first signal of the LSP, the second ODU1 label corresponds to the second signal of the LSP, etc. For instance, the corresponding labels can take the following values:

- First ODU1: $t 3=2, \quad t 2=0, t 1=0$ (in first TS of ODTUG3)

- Second ODU1: $t 3=10, t 2=0, t 1=0$ (in ninth TS of ODTUG3)

- Third ODU1: $t 3=7, t 2=0, t 1=0$ (in sixth TS of ODTUG3)

- Fourth ODU1: $t 3=6, t 2=0, t 1=0$ (in fifth TS of ODTUG3) 


\section{RSVP-TE Signaling Protocol Extensions}

This section specifies the [RFC3473] protocol extensions needed to accommodate G.709 traffic parameters.

The G.709 traffic parameters are carried in the G.709 SENDER_TSPEC and FLOWSPEC objects. The same format is used both for SENDER_TSPEC object and FLOWSPEC objects. The content of the objects is defined above in section 3.2. The objects have the following class and type for G.709:

- G.709 SENDER_TSPEC Object: Class $=12, \mathrm{C}-\mathrm{Type}=5$

- G.709 FLOWSPEC Object: Class $=9$, C-Type $=5$

There is no Adspec associated with the G.709 SENDER_TSPEC. Either the Adspec is omitted or an Int-serv Adspec with the Default General Characterization Parameters and Guaranteed Service fragment is used, see [RFC2210].

For a particular sender in a session, the contents of the FLOWSPEC object received in a Resv message SHOULD be identical to the contents of the SENDER_TSPEC object received in the corresponding Path message. If the objects do not match, a Resverr message with a "Traffic Control Error/Bad Flowspec value" error SHould be generated.

Intermediate and egress nodes MUST verify that the node itself, and the interfaces on which the LSP will be established, can support the requested Signal Type, NMC, and NVC values (as defined in section 3.2). If the requested value(s) cannot be supported, the receiver node MUST generate a PathErr message with a "Traffic Control Error/Service unsupported" indication (see [RFC2205]).

In addition, if the $\mathrm{MT}$ field is received with a zero value, the node MUST generate a PathErr message with a "Traffic Control Error/Bad Tspec value" indication (see [RFC2205]).

7. Security Considerations

This document introduces no new security considerations to [RFC3473].

8. IANA Considerations

Two values have been defined by IANA for this document:

Two RSVP C-Types in registry:

http://www.iana.org/assignments/rsvp-parameters 
- A G.709 SENDER_TSPEC object: Class $=12$, C-Type $=5$ - see Section 6 .

- A G.709 FLOWSPEC object: Class $=9$, C-Type $=5$ - see Section 6 .

IANA will also track the code-point spaces extended and/or updated by this document. For this purpose, the following new registry entries have been added in the newly requested registry entry: http://www.iana.org/assignments/gmpls-sig-parameters

- LSP Encoding Type:

Name: LSP Encoding Type

Format: 8-bit number Values:
[1..11]
defined in [RFC3471]
12
13
defined in section 3.1.1
defined in Section 3.1.1

Allocation Policy:
[0..239]
Assigned by IANA via IETF Standards Track RFC
$[240 . .255]$
Action.
Assigned temporarily for Experimental Usage.
These will not be registered with IANA

- Switching Type:

Name: Switching Type

Format: 8-bit number

Values: defined in [RFC3471]

Allocation Policy:
[0..255]
Assigned by IANA via IETF Standards Track RFC Action.

- Generalized PID (G-PID) :

Name: G-PID

Format: 16-bit number

Values:

[0..31] defined in [RFC3471]

[32..35] defined in [RFC3471] and updated by section

3. 1.3

$[36 . .46]$

defined in [RFC3471]

[47..58]

defined in section 3.1.3

Allocation Policy:

[0..31743] Assigned by IANA via IETF Standards Track RFC Action.

[31744..32767] Assigned temporarily for Experimental Usage 
[32768.65535] Not assigned. Before any assignments can be made in this range, there MUST be a standards Track RFC that specifies IANA Considerations that covers the range being assigned.

Note: per [RFC3471], Section 3.1.1, standard Ethertype values are used as G-PIDs for packet and Ethernet LSPs.

\section{Acknowledgements}

The authors would like to thank Jean-Loup Ferrant, Mathieu Garnot, Massimo Canali, Germano Gasparini, and Fong Liaw for their constructive comments and inputs as well as James Fu, Siva Sankaranarayanan, and Yangguang Xu for their useful feedback. Many thanks to Adrian Farrel for having thoroughly reviewed this document.

This document incorporates (upon agreement) material and ideas from a work in progress, "Common Label and Label Request specification for Automatic Switched Transport Network", by Zhi Lin.

10. References

10.1. Normative References

[RFC2119] Bradner, S., "Key words for use in RFCs to Indicate Requirement Levels", BCP 14, RFC 2119, March 1997.

[RFC2205] Braden, R., Zhang, L., Berson, S., Herzog, S., and S. Jamin, "Resource ReSerVation Protocol (RSVP) -- Version 1 Functional Specification", RFC 2205, september 1997.

[RFC2210] Wroclawski, J., "The Use of RSVP with IETF Integrated Services", RFC 2210, september 1997.

[RFC3471] Berger, L., "Generalized Multi-Protocol Label Switching (GMPLS) Signaling Functional Description", RFC 3471, January 2003 .

[RFC3473] Berger, L., "Generalized Multi-Protocol Label Switching (GMPLS) Signaling Resource ReserVation Protocol-Traffic Engineering (RSVP-TE) Extensions", RFC 3473, January 2003 .

[RFC3946] Mannie, E. and D. Papadimitriou, "Generalized MultiProtocol Label Switching (GMPLS) Extensions for Synchronous Optical Network (SONET) and Synchronous Digital Hierarchy (SDH) Control", RFC 3946, October 2004 . 
[RFC4202] Kompella, K., Ed. and Y. Rekhter, Ed., "Routing Extensions in Support of Generalized Multi-Protocol Label Switching (GMPLS)", RFC 4202, September 2005.

10.2. Informative References

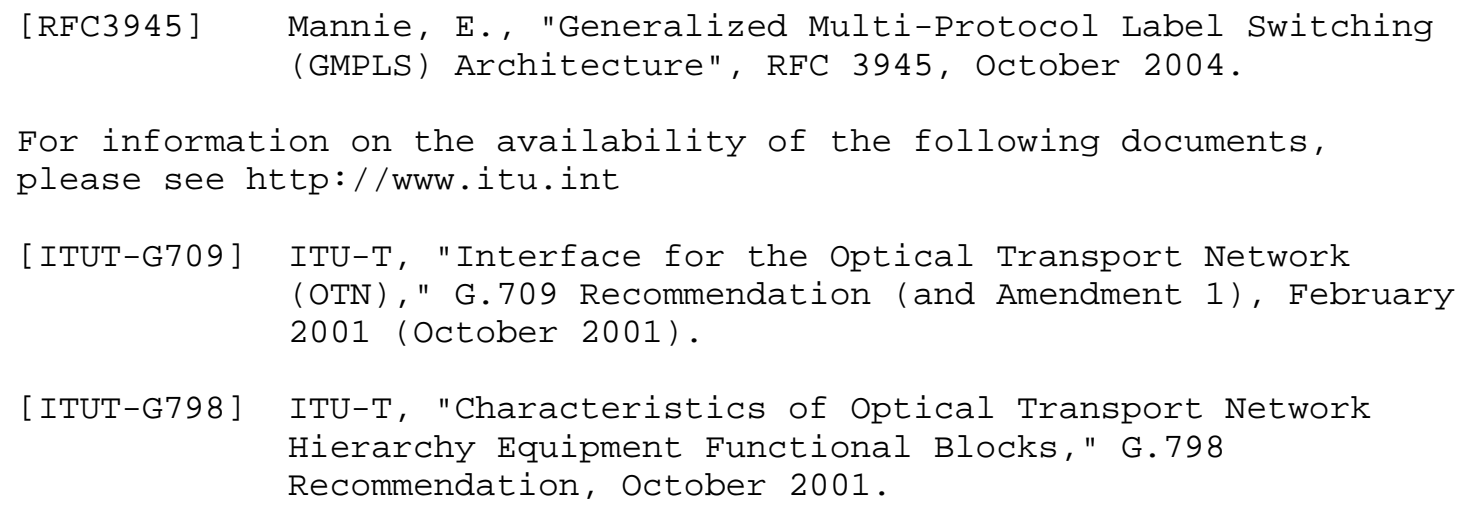

11. Contributors

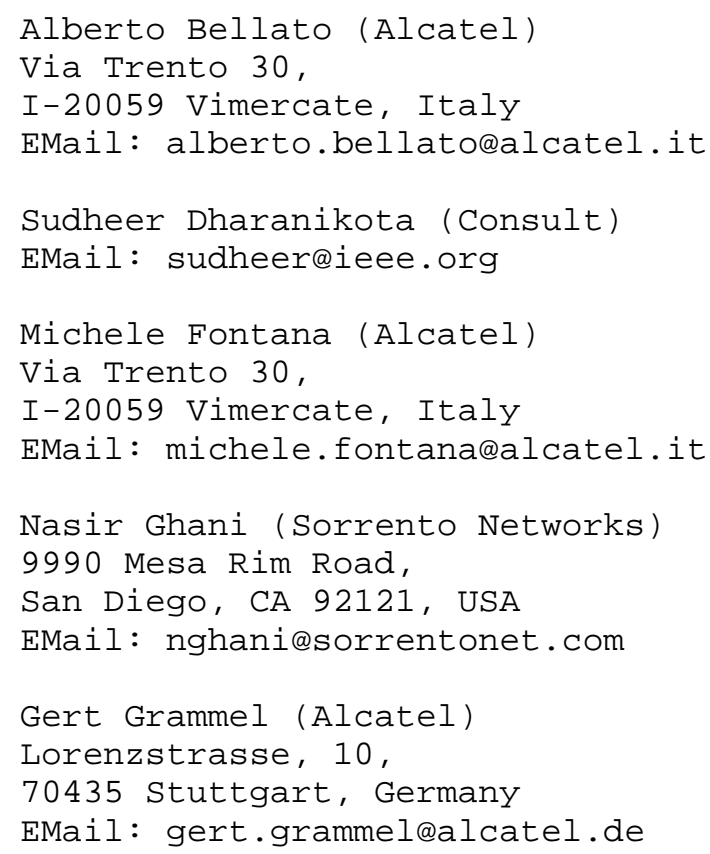




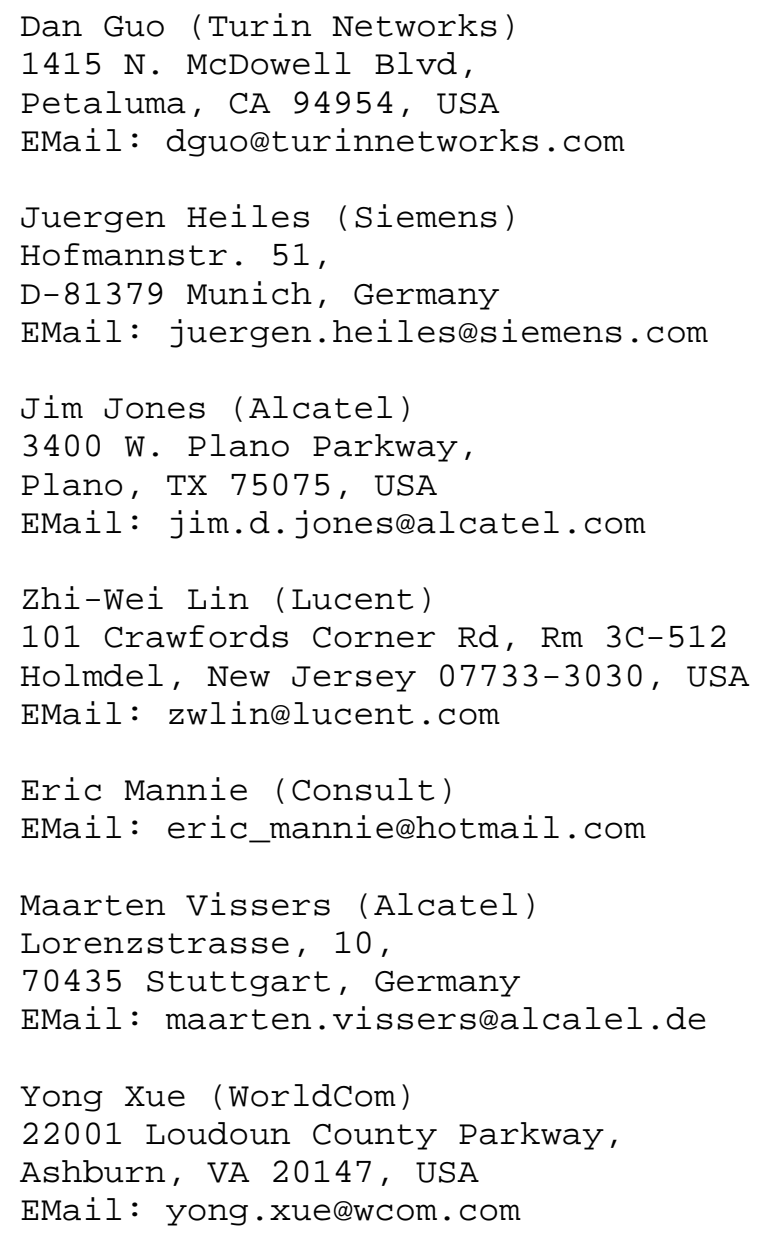


Appendix A. Abbreviations

\begin{tabular}{|c|c|}
\hline BSNT & Bit Stream without Octet Timing \\
\hline BSOT & Bit Stream with Octet Timing \\
\hline CBR & Constant Bit Rate \\
\hline ESCON & Enterprise Systems Connection \\
\hline $\mathrm{FC}$ & Fiber Channel \\
\hline FEC & Forward Error Correction \\
\hline FICON & Fiber Connection \\
\hline FSC & Fiber Switch Capable \\
\hline GCC & General Communication Channel \\
\hline GFP & Generic Framing Procedure \\
\hline LSC & Lambda Switch Capable \\
\hline LSP & Label Switched Path \\
\hline MS & Multiplex Section \\
\hline $\mathrm{naOH}$ & non-associated Overhead \\
\hline NMC & Number of Multiplexed Components \\
\hline NVC & Number of Virtual Components \\
\hline OCC & Optical Channel Carrier \\
\hline OCG & Optical Carrier Group \\
\hline $\mathrm{OCh}$ & Optical Channel (with full functionality) \\
\hline OChr & Optical Channel (with reduced functionality) \\
\hline ODTUG & Optical Date Tributary Unit Group \\
\hline ODU & Optical Channel Data Unit \\
\hline $\mathrm{OH}$ & Overhead \\
\hline OMS & Optical Multiplex Section \\
\hline $\mathrm{OMU}$ & Optical Multiplex Unit \\
\hline OOS & OTM Overhead Signal \\
\hline OPS & Optical Physical Section \\
\hline OPU & Optical Channel Payload Unit \\
\hline OSC & Optical Supervisory Channel \\
\hline $\mathrm{OTH}$ & Optical Transport Hierarchy \\
\hline OTM & Optical Transport Module \\
\hline OTN & Optical Transport Network \\
\hline OTS & Optical Transmission Section \\
\hline OTU & Optical Channel Transport Unit \\
\hline OTUkV & Functionally standardized OTUk \\
\hline $\mathrm{PPP}$ & Point to Point Protocol \\
\hline PSC & Packet Switch Capable \\
\hline RES & Reserved \\
\hline $\mathrm{RS}$ & Regenerator Section \\
\hline TTI & Trail Trace Identifier \\
\hline TDM & Time Division Multiplex \\
\hline
\end{tabular}


Appendix B. G.709 Indexes

- Index k: The index "k" is used to represent a supported bit rate and the different versions of OPUk, ODUk and OTUk. k=1 represents an approximate bit rate of 2.5 Gbit/s, $k=2$ represents an approximate bit rate of $10 \mathrm{Gbit} / \mathrm{s}, \mathrm{k}=3$ an approximate bit rate of $40 \mathrm{Gbit} / \mathrm{s}$ and $\mathrm{k}=$ 4 an approximate bit rate of $160 \mathrm{Gbit} / \mathrm{s}$ (under definition). The exact bit-rate values are in $\mathrm{kbits} / \mathrm{s}$ :

- OPU: k=1: $2488320.000, k=2: 9995276.962, k=3: 40150519.322$

- ODU: $k=1: 2498775.126, k=2: 10037273.924, k=3: 40319218.983$

- OTU: k=1: $2666057.143, k=2: 10709225.316, k=3: 43018413.559$

- Index m: The index "m" is used to represent the bit rate or set of bit rates supported on the interface. This is a one or more digit "k", where each "k" represents a particular bit rate. The valid values for $m$ are $(1,2,3,12,23,123)$.

- Index n: The index "n" is used to represent the order of the OTM, OTS, OMS, OPS, OCG and OMU. This index represents the maximum number of wavelengths that can be supported at the lowest bit rate supported on the wavelength. It is possible that a reduced number of higher bit rate wavelengths are supported. The case $\mathrm{n}=0$ represents a single channel without a specific wavelength assigned to the channel.

- Index r: The index "r", if present, is used to indicate a reduced functionality OTM, OCG, OCC and OCh (non-associated overhead is not supported). Note that for $n=0$ the index $r$ is not required as it implies always reduced functionality.

Editor's Address

Dimitri Papadimitriou (Alcatel)

Francis Wellesplein 1,

B-2018 Antwerpen, Belgium

Phone: +32 3 240-8491

EMail: dimitri.papadimitrioulalcatel.be 
Full Copyright statement

Copyright (C) The Internet Society (2006).

This document is subject to the rights, licenses and restrictions contained in BCP 78, and except as set forth therein, the authors retain all their rights.

This document and the information contained herein are provided on an "AS IS" basis and THE CONTRIBUTOR, THE ORGANIZATION HE/SHE REPRESENTS OR IS SPONSORED BY (IF ANY), THE INTERNET SOCIETY AND THE INTERNET ENGINEERING TASK FORCE DISCLAIM ALL WARRANTIES, EXPRESS OR IMPLIED, INCLUDING BUT NOT LIMITED TO ANY WARRANTY THAT THE USE OF THE INFORMATION HEREIN WILL NOT INERINGE ANY RIGHTS OR ANY IMPLIED WARRANTIES OF MERCHANTABILITY OR FITNESS FOR A PARTICULAR PURPOSE.

Intellectual Property

The IETF takes no position regarding the validity or scope of any Intellectual property Rights or other rights that might be claimed to pertain to the implementation or use of the technology described in this document or the extent to which any license under such rights might or might not be available; nor does it represent that it has made any independent effort to identify any such rights. Information on the procedures with respect to rights in RFC documents can be found in BCP 78 and BCP 79 .

Copies of IPR disclosures made to the IETF Secretariat and any assurances of licenses to be made available, or the result of an attempt made to obtain a general license or permission for the use of such proprietary rights by implementers or users of this specification can be obtained from the IETF on-line IPR repository at http://www.ietf.org/ipr.

The IETF invites any interested party to bring to its attention any copyrights, patents or patent applications, or other proprietary rights that may cover technology that may be required to implement this standard. Please address the information to the IETF at ietf-ipreietf.org.

Acknowledgement

Funding for the RFC Editor function is provided by the IETF Administrative Support Activity (IASA) . 